\title{
METHICILLIN-RESISTANT STAPHYLOCOCCI PREVALENCE IN CANCER PATIENTS AT A TERTIARY CARE CANCER CENTRE - A RETROSPECTIVE STUDY
}

\author{
SARAVANAN MURUGESAN ${ }^{1}$, SUJINA TK ${ }^{2}$, SAJANI SAMUEL ${ }^{1}$, SARATH KE ${ }^{1}$, PARTHIBAN RUDRAPATHY ${ }^{1 *}$ \\ ${ }^{1}$ Division of Microbiology, Department of Clinical Laboratory Services and Translational Research (CLS \& TR), Malabar Cancer Centre, \\ Thalassery, Kannur, Kerala, India. ${ }^{2}$ Palayad Campus, Kannur University, Kerala, India. Email: parthi71975@gmail.com
}

Received: 08 March 2021, Revised and Accepted: 30 April 2021

\section{ABSTRACT}

Objective: The objective of this study was to investigate the prevalence of Methicillin-resistant Staphylococcus aureus (MRSA) and MR-coagulasenegative staphylococci (CoNS), as well as their antimicrobial resistance, in various samples from cancer patients in North Kerala.

Methods: The retrospective study was conducted at a tertiary care cancer centre in North Kerala over a 4-year period from January 2016 to December 2019. During the study, data on all cultures from cancer patients was analyzed. This study was approved by Institutional Review Board (IRB). Nonduplicate isolates of staphylococci were included in the study obtained from various clinical specimens. Species identification and antimicrobial susceptibility testing was done using automated methods.

Results: During the period of 4 years (2016-2019), a total of 1176 isolates of staphylococci were analyzed, out of which 784 were S. aureus isolates $(68 \%)$ and $392(32 \%)$ isolates were CoNS. Among CoNS species, Staphylococcus epidermidis and Staphylococcus haemolyticus were the most common species of CoNS, representing 39\% and $28 \%$ of the total CoNS identified. Overall prevalence of methicillin resistance in S. aureus and CoNS was found to be $50.7 \%$ and $55.6 \%$, respectively. Methicillin-resistant staphylococci isolates showed higher resistance to multiple drugs than methicillin-sensitive staphylococci isolates.

Conclusion: This study demonstrates that MRS could also be a haul in cancer patients at North Kerala. A higher percentage of MR-CoNS isolates are multidrug resistant than MRSA isolates. Glycopeptides and linezolid still stay the mainstay for treatment for MRS infections.

Keywords: Methicillin-resistant staphylococci, Prevalence, Antibiotic resistance, Cancer patients.

(C) 2021 The Authors. Published by Innovare Academic Sciences Pvt Ltd. This is an open access article under the CC BY license (http://creativecommons.org/ licenses/by/4.0/) DOI: http://dx.doi.org/10.22159/ajpcr.2021v14i7.41482. Journal homepage: https://innovareacademics.in/journals/index.php/ajpcr

\section{INTRODUCTION}

Infections are common among cancer patients, lengthening hospital stays, and increasing health-care costs. Nosocomial infections account for the majority of infections in cancer patients [1,2]. Staphylococcus is a genus of bacteria that causes a wide range of infectious diseases in humans. It is divided into two types: S. aureus and coagulasenegative staphylococci (CoNS). CoNS are opportunistic pathogens that are generally associated with infections in patients that have medical devices or who are immunocompromised [3-6]. Among the CoNS, Staphylococcus epidermidis and Staphylococcus haemolyticus are much more virulent than the other CoNS species [4-6].

Methicillin-resistant staphylococci (MRS) are increasingly being classified as multidrug-resistant (MDR) due to their high resistance to a wide range of commonly used antimicrobials such as erythromycin, clindamycin, aminoglycosides, fluoroquinolones, and antibiotic medication, leaving few effective options. MDR among CoNS is on the rise, posing a significant challenge in the management of hospital-acquired infections and acting as a reservoir of antibiotic resistance [5,7].

There is no information available on the prevalence of Methicillinresistant $S$. aureus (MRSA) and Methicillin-resistant CoNS (MR-CoNS) in cancer patients in Southern India. As a result, the current research was undertaken to investigate the prevalence of MRSA and MR-CoNS, as well as their antimicrobial resistance, in various samples from cancer patients in North Kerala.

\section{METHODS}

This retrospective study was conducted at a tertiary care cancer center at North Kerala, India. January 2016-December 2019 data on all microbial cultures from various clinical samples from cancer patients were analyzed during the period - February 2020-May 2020. This study was approved by Institutional Review Board (IRB) [1616/IRBSRC/13/MCC/14-03-2020/4].

The study included only non-duplicate isolates of S. aureus and CoNS (clinically significant) was included in the study. The isolates were considered to be of clinical significance only when the following criteria were fulfilled: (1) Strains obtained in bacterial pure culture; (2) infection risk factors (immunosuppressive); (3) clinical symptoms of infection (hyperthermia $>38^{\circ} \mathrm{C}$, hypotension, tachycardia, tachypnea); and (4) infection site (respiratory, intestinal, joints, skin, and soft tissues).

These staphylococci species were isolated from varied clinical samples such as pus, sputum, urine, blood, and body fluids. Blood agar, MacConkey agar, and chocolate agar plates were used to culture the clinical specimens, which were incubated aerobically at $37^{\circ} \mathrm{C}$ for 24-48 h. Bactec 9050 (BD BactecTM 9050 Blood Culture System) and BacT/ALERT 3D (Biomerieux, USA) were used to process blood cultures according to the manufacturer's instructions.

Bacterial identification and Antibiotic susceptibility testing (AST) After the initial colony isolation from the culture plates, these colonies were then subjected for species identification by automated identification system (Phoenix and Vitek 2 automated system) and from 2019 onward all the staphylococci were identified using Matrix-assisted laser desorption ionization time flight mass-spectroscopy (MALDI-TOFMS - Biomerieux, France). After identification of staphylococcal species, antibiotic susceptibility was performed using automated system. These colonies were taken from the plate and suspension was made 
and compared with the MacFarland standard before inoculating into the AST panel kits. AST was performed using BD PhoenixTM (Becton Dickinson, USA) instrument from January 2016 to October 2017 and thereafter using VITEK 2 Compact system (Biomerieux, France). The bacterial suspensions were added to the commercially available Grampositive kit (GPC- P628) and loaded into the automated system for AST.

AST of those isolates was performed against a panel of antimicrobials. This automated system monitors the kinetics of bacterial growth, calculates it using a unique algorithm and follows a Clinical and Laboratory Standards Institute (CLSI) guidelines [8]. As a quality control, S. aureus ATCC 25923 was used.

\section{Statistical analysis}

WHONET 5.6 software was used to evaluate the isolated staphylococcal species and its antibiotic susceptibility pattern. Using SPSS version 21.0 software, a Chi-square test for linear trend was used to compare antibiotic resistance patterns (SPSS Inc., Chicago, IL, USA). A statistically relevant $\mathrm{p}$ value was $<0.05$

\section{RESULTS}

A total of 1176 isolates of staphylococci were analyzed (Table 1). The skin and soft tissue infections (SSTI) (60\%) in addition as lower respiratory tract $(23 \%)$ were the most important sources of S. aureus isolates. On the other hand, majority of the CoNS were from SSTI (54\%), followed by blood (25\%) and lower respiratory tract (17\%) (Table 2). Gender-wise distribution (male and female) among $S$. aureus was found to be 1:08, whereas CoNS is significantly higher in men population (1:06) than women.

All staphylococcal species were identified using automated methods. Among CoNS species, Staphylococcus epidermidis $(\mathrm{n}=351 ; 39 \%)$ was the predominant species, followed by $S$. haemolyticus $(\mathrm{n}=111 ; 28 \%)$, S. hominis $(\mathrm{n}=49 ; 12 \%)$, S. saprophyticus $(\mathrm{n}=22 ; 6 \%)$, and other CoNS species $(\mathrm{n}=59 ; 15 \%)$.

The sensitivity of all staphylococci to widely used antibiotics was tested. The prevalence of MRSA and MR-CoNS during the study period is given in Figs. 1 and 2. Overall prevalence of MRSA and MR-CoNS was found to be $50.7 \%$ and $55.6 \%$, respectively. Our study shows increasing trend in MRS infection among cancer patients from 2016 to 2019. The prevalence of MRSA isolates was observed from 2016 (37\%) with 2018 (58\%) and 2019 (57.5\%) showing a significant rise in MRSA incidence. An alarming upward trend in MR-CoNS when compared to MRSA. The prevalence of MR-CoNS strains was observed from $2016(20 \%)$ to 2019 (87.5\%) showing a significant rise in MR-CoNS incidence.

In the present study, MRSA and MR-CoNS represent variability in drug-resistant pattern. MRSA isolates revealed the highest resistance to ciprofloxacin (84\%), followed by erythromycin (79\%) and clindamycin (27\%), whereas MR-CoNS isolates showed highest resistance to co-trimoxazole (77\%), followed by erythromycin (69\%) and ciprofloxacin (67\%). About $1 \%$ and $2.3 \%$ of MRSA and MRCoNS isolates were found to be resistant to linezolid, respectively. All the isolates of staphylococci were sensitive to glycopeptides (Figs. 3 and 4). The incidence of inducible (iMLS ) and constitutive $\left(\mathrm{CMLS}_{\mathrm{B}}\right)$ clindamycin resistance was higher in MRSA (iMLS $\mathrm{B}, 17 \%$; $\mathrm{CMLS}_{\mathrm{B}}-6.2 \%$ ) than MSSA (iMLS, $12.7 \%$ c $_{\mathrm{B}}$ MLS $\left.-1.3 \%\right)$; whereas among MR-CoNS (iMLS $16 \%$; $\mathrm{CMLS}_{\mathrm{B}}-27.5 \%$ ) and MS-CoNS (iMLS $\mathrm{B}_{\mathrm{B}} 3.4 \%$ cMLS $_{\mathrm{B}}-1.7 \%$ ) (Table 3 ).

\section{DISCUSSION}

MRS have disseminated worldwide and still be among the foremost common hospital pathogens. The prevalence and characterization of MRSA and MR-CoNS in hospitals are reported from different parts of the Globe [3,5,9-15]. In the present study, 4-year data of staphylococci infections in cancer patients and prevalence of MRS and

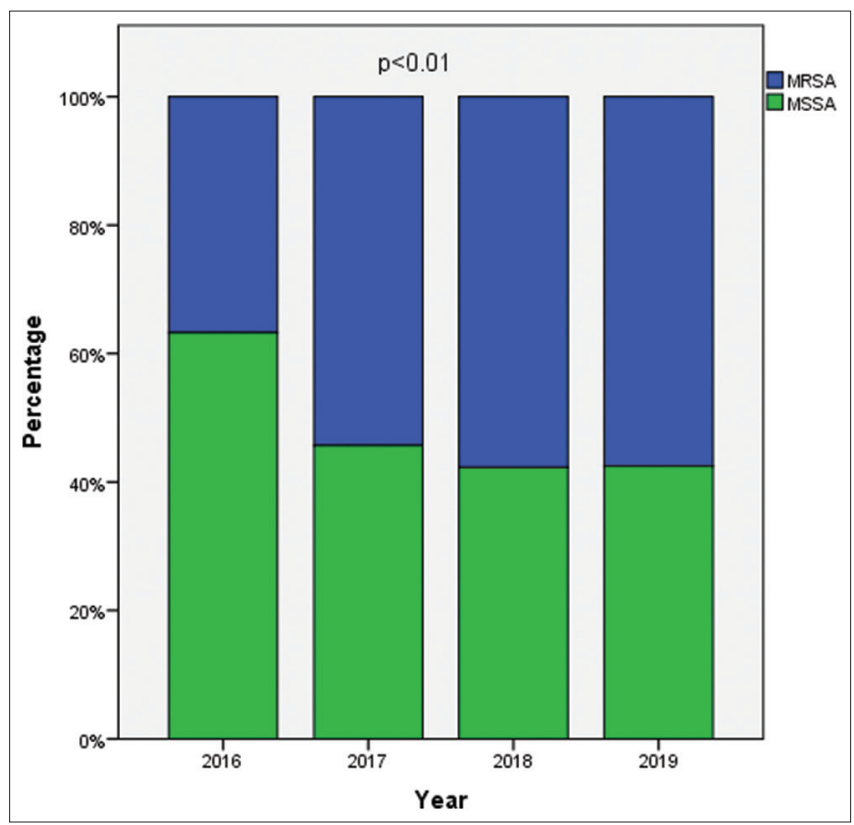

Fig. 1: Prevalence of methicillin-resistant Staphylococcus aureus (MRSA) and methicillin-sensitive Staphylococcus aureus (MSSA)

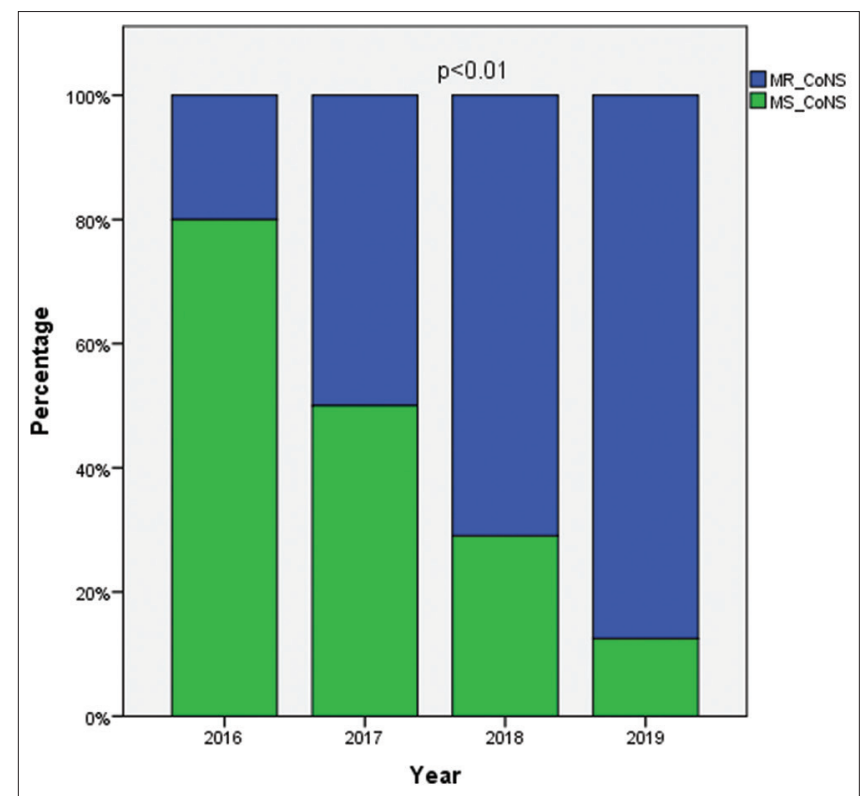

Fig. 2: Prevalence of methicillin-resistant coagulase-negative staphylococci (MR-CoNS) and methicillin-sensitive coagulasenegative staphylococci (MS-CoNS)

Table 1: Overall prevalence of staphylococci in our hospital

\begin{tabular}{llllll}
\hline Staphylococci & $\mathbf{2 0 1 6}(\mathbf{n}=\mathbf{3 2 4})$ & $\mathbf{2 0 1 7}(\mathbf{n}=\mathbf{2 5 7})$ & $\mathbf{2 0 1 8}(\mathbf{n}=\mathbf{3 9 4})$ & $\mathbf{2 0 1 9}(\mathbf{n}=\mathbf{2 0 1})$ & Total prevalence $\mathbf{n}(\mathbf{\%})$ \\
\hline Staphylococcus aureus & 234 & 151 & 246 & 153 & $784(66.7)$ \\
CoNS & 90 & 106 & 148 & 48 & $392(33.3)$ \\
\hline
\end{tabular}

CoNS: Coagulase-negative staphylococci 
Table 2: Sample-wise distribution of methicillin- sensitive staphylococci and methicillin-resistant staphylococci

\begin{tabular}{lllll}
\hline $\begin{array}{l}\text { Type of } \\
\text { specimen }\end{array}$ & $\begin{array}{l}\text { Staphylococcus } \\
\text { aureus } \\
\text { n (\%) }\end{array}$ & $\begin{array}{l}\text { MRSA } \\
\mathbf{n}(\%)\end{array}$ & $\begin{array}{l}\text { CoNS } \\
\mathbf{n}(\%)\end{array}$ & $\begin{array}{l}\text { MR-CoNS } \\
\mathbf{n}(\%)\end{array}$ \\
\hline Blood & $59(7.5)$ & $36(9)$ & $97(25)$ & $42(19)$ \\
Respiratory & $182(23)$ & $105(26)$ & $68(17)$ & $40(18)$ \\
Exudates & $468(60)$ & $224(56)$ & $213(54)$ & $133(61)$ \\
Urine & $21(3)$ & $11(3)$ & $12(3)$ & $2(1)$ \\
Body fluids & $54(7)$ & $22(6)$ & - & - \\
\hline
\end{tabular}

Table 3: Prevalence of inducible and constitutive clindamycin resistance among methicillin-sensitive staphylococci and methicillin-resistant staphylococci

\begin{tabular}{lllll}
\hline Resistant phenotype & $\begin{array}{l}\text { MSSA } \\
\text { n (\%) }\end{array}$ & $\begin{array}{l}\text { MRSA } \\
\text { n (\%) }\end{array}$ & $\begin{array}{l}\text { MS-CoNS } \\
\text { n (\%) }\end{array}$ & $\begin{array}{l}\text { MR-CoNS } \\
\text { n (\%) }\end{array}$ \\
\hline${ }_{\mathrm{i}} \mathrm{MLS}_{\mathrm{B}}$ & $48(13)$ & $68(17)$ & $6(3.4)$ & $35(16)$ \\
$\mathrm{CMLS}_{\mathrm{B}}$ & $5(1.3)$ & $25(6.2)$ & $3(1.7)$ & $70(27.5)$ \\
\hline
\end{tabular}

MLSB: Macrolide-Lincosamide-StreptograminB

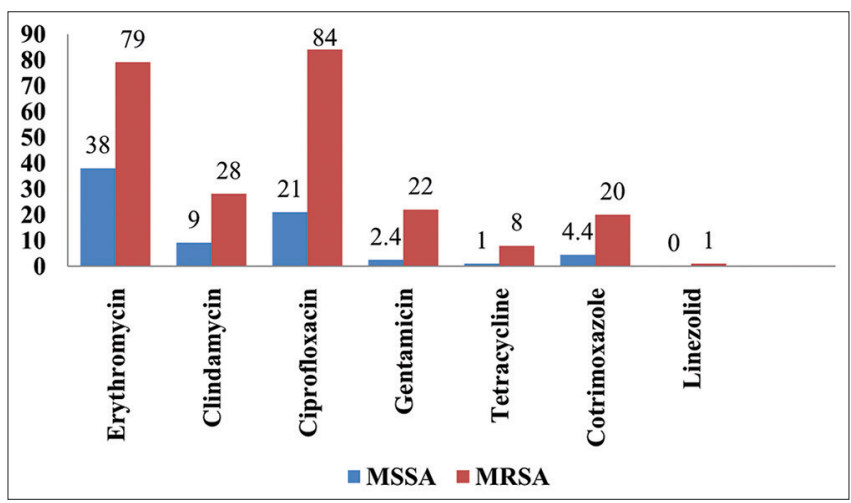

Fig. 3: Overall resistance pattern among methicillin-sensitive $S$. aureus and methicillin-resistant $S$. aureus isolates

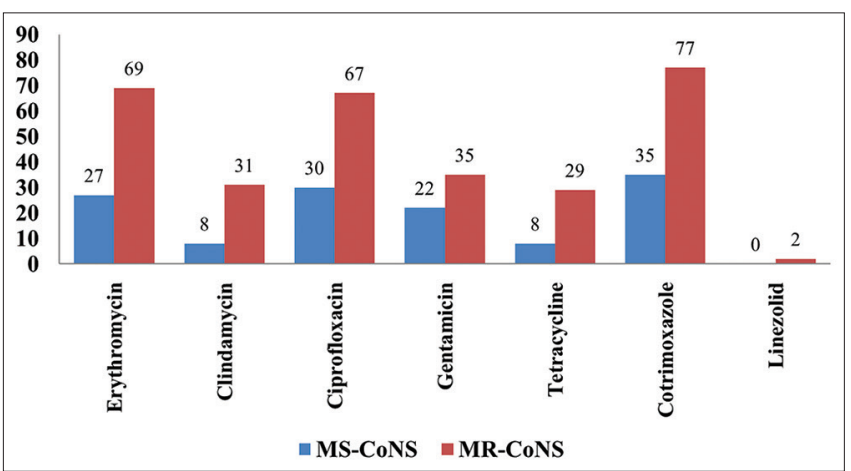

Fig. 4: Overall resistance pattern among methicillin-sensitive coagulase-negative staphylococci (MS-CoNS) and methicillinresistant coagulase-negative staphylococci (MR-CoNS)

antibiotic susceptibility were analyzed. The incidence of MRS strains was evaluated in several cultures obtained from different cancer patient specimens. In the present study, around $60 \%$ of the staphylococci isolates were from exudates, followed by respiratory specimens and Blood. This finding was consistent with the previous reports from India and worldwide $[11,16,17]$.

In our study, the overall prevalence of MRSA was $50.7 \%$. The observed resistance was very low in 2016 (37\%) in comparison to
2019 (57.5\%). In our study, we found higher rate of MRSA (50.7\%) when compared to the previous multicenter studies from India $[10,13]$. On the contrary, some studies $[12,18,19]$ have found an alarmingly high incidence of MRSA in India and around the world. MRSA prevalence in India and elsewhere, which ranged from $21 \%$ to $82 \%$, may be due to regional differences in study design, sample population, antibiotic policies, and thus infection control steps.

MDR patterns were more common in MRSA than MSSA. MRSA isolates showed higher resistance to ciprofloxacin, erythromycin, and clindamycin, though comparable rates of resistance were noticed in gentamicin and co-trimoxazole. The high and increasing level of resistance to ciprofloxacin, a routine antimicrobial, altogether the hospitals was may be expected due to its pervasive use. The findings throughout this study was according to the previous multicentric studies $[11,20]$ and which was not concordance to the previous report [18]. Clindamycin is an efficient drug for staphylococcal skin and soft-tissue infections. Resistance to the macrolide group of antibiotics in staphylococci has been reported from different parts of the world. The overall prevalence of inducible clindamycin resistance among MRSA and MSSA isolates was $68(17.5 \%)$ and $48(12 \%)$, respectively. This was not according to the previous studies performed in South-India and worldwide $[15,18]$. In general, it is going to be risky to use clindamycin once erythromycin testing shows resistance or intermediate although the microorganism is sensitive to clindamycin. For this reason, routine D-testing may facilitate clinicians to retain.

The alarming increase in MR-CoNS is limiting the effectiveness of all $\beta$-lactam agents, limiting therapeutic options significantly. Linezolid could also be a viable solution for methicillin resistant staphylococcal species. According to multinational and multicenter surveillance studies, linezolid is effective against $99 \%$ of $S$. aureus and CoNS clinical strains $[5,10,11,20]$. Emerging resistance to linezolid, on the other hand, may be a major source of concern. It is time that we tend to acknowledge the way reaching consequences expose by such an excellent threat and closely monitor and track resistance to linezolid by prospective resistance surveillance studies, notably wherever frequent and extended linezolid therapy is employed.

MR-CoNS are one of the most common causes of human infections, and their MDR property is more noticeable than that of MS-CoNS. Further, it also serves as a reservoir for resistance genes. Among CoNS, $S$. epidermidis and $S$. haemolyticus were the predominant species reported. The identification of CoNS was performed using automated methods also as MALDI-TOF.

The species distribution of CoNS in our study was concordance with the previous study from India where S. epidermidis (39\%) was the predominant species, followed by $S$. haemolyticus $(28 \%)[9,10,16]$. In contrast, few studies from India and worldwide reported S. haemolyticus as the most common species, followed by $S$. epidermidis $[3,11,13]$. The varying adaptability of different organisms to selective pressures such as biocides and antimicrobials within the ecosystem may be due to differences in colonization characteristics of patients in India and around the world.

Many reports have found a rise in the incidence of hospital-acquired infections caused by MR-CoNS strains in recent years. According to the surveys, the prevalence of MR-CoNS was higher (75-90\%) than MRSA in the 1990s, and this trend continues today [4,5]. This was reflected within the present study, MR-CoNS (55.6\%) displayed higher percentage in comparison to MRSA (50.7\%). Furthermore, MR-CoNS showed high resistance to other non- $\beta$-lactam antimicrobial agents co-trimoxazole (77\%), erythromycin (69\%), and ciprofloxacin (67\%). An overall high prevalence of resistance to all or any antibiotics was observed with MR-CoNS - showing higher resistance to other non $\beta$-lactam antimicrobial agents as compared to MS-CoNS. Within the present study, the general incidence of inducible and constitutive $\mathrm{MLS}_{\mathrm{B}}$ resistance phenotype among the CoNS isolates was $10 \%$ and $19 \%$, 
respectively. This is often not supported by other studies from India and worldwide $[5,10]$.

As a result, routine monitoring of hospital-associated infections, as well as the antimicrobial susceptibility pattern of MRS and the formulation of a specific antibiotic strategy, may help reduce the burden of MRS infections within the hospital.

\section{CONCLUSION}

This study demonstrates that MRS may be a problem in cancer patients at North Kerala. A higher percentage of MR-CoNS isolates are MDR than MRSA isolates. Glycopeptides and linezolid still remain the mainstay for treatment for MRS infections. To reduce the prevalence of nosocomial infection in critical care areas, we recommend education and awareness among healthcare workers and clinicians, as well as adherence to simple guidelines for prevention of nosocomial infection.

Hand hygiene is also the most important tool for preventing infection in health-care settings. This current study will aid our hospital to educate the employees using their own data to drive home the value of their hand hygiene. This study's findings can also be used to help us refocus approaches in other fields.

\section{ACKNOWLEDGMENT}

Nil.

\section{AUTHORS' CONTRIBUTIONS}

Dr. Saravanan M, Ms. Sujina TK, and Dr. Parthiban R contributed substantially to the conception, design of the study, analysis, and interpretation of data. All authors discussed the results and commented on the manuscript. Dr. Saravanan M and Dr. Parthiban R drafted the final manuscript.

\section{CONFLICT OF INTEREST STATEMENT}

None to declare.

\section{FUNDING SOURCES}

Nil.

\section{REFERENCES}

1. Bhat V, Gupta S, Kelkar R, Biswas S, Khattry N, Moiyadi A, et al. Bacteriological profile and antibiotic susceptibility patterns of clinical isolates in a tertiary care cancer center. Indian J Med Paediatr Oncol 2016;37:20-4

2. El-Gendy MM, El-Bondkly AM, Keera AA, Ali AM. Incidence of methicillin resistant Staphylococcus aureus (MRSA) in microbial community of cancer patients and evaluation of their resistant pattern. Arab J Sci Eng 2017;43:83-92.

3. Kitti T, Seng R, Saiprom N, Thummeepak R, Chantratita N, Boonlao C, et al. Molecular characteristics of methicillin-resistant staphylococci clinical isolates from a tertiary Hospital in Northern Thailand. Can J Infect Dis Med Microbiol 2018;2018:8457012.

4. Becker K, Both A, Weißelberg S, Heilmann C, Rohde H. Emergence of coagulase-negative staphylococci. Expert Rev Anti Infect Ther 2020;18:349-6.
5. Montazeri EA, Seyed-Mohammadi S, Dezfuli AA, Khosravi AD, Dastoorpoor M, Roointan M, et al. Investigation of SCCmec types I-IV in clinical isolates of methicillin-resistant coagulasenegative staphylococci in Ahvaz, Southwest Iran. Biosci Rep 2020;40:BSR20200847.

6. Nanoukon C, Argemi X, Sogbo F, Orekan J, Keller D, Affolabi D, et al. Pathogenic features of clinically significant coagulase-negative staphylococci in hospital and community infections in Benin. Int J Med Microbiol 2017;307:75-82

7. McManus BA, Coleman DC, Deasy EC, Brennan GI, O’Connell B, Monecke $\mathrm{S}$, et al. Comparative genotypes, staphylococcal cassette chromosome mec (SCCmec) genes and antimicrobial resistance amongst Staphylococcus epidermidis and Staphylococcus haemolyticus isolates from infections in humans and companion animals. PLoS One 2015; 10:e0138079.

8. Clinical and Laboratory Standards Institute. Performance Standards for Antimicrobial Susceptibility Testing. Vol. 30. Wayne, PA: Clinical and Laboratory Standards Institute; 2014.

9. Murugesan S, Perumal N, Dass BS, Vijayakumar R, Krishnan P. Prevalence and molecular characterisation of methicillin-resistant coagulase negative staphylococci (MR-CoNS) isolated from nasal carriers of end stage renal disease patients-a prospective study. J Clin Diagn Res 2019;13:DC10-5.

10. Bora P, Datta P, Gupta V, Singhal L, Chander J. Characterization and antimicrobial susceptibility of coagulase-negative staphylococci isolated from clinical samples. J Lab Physicians 2018;10:414-9.

11. Rajkumar S, Sistla S, Manoharan M, Sugumar M, Nagasundaram N, Parija SC, et al. Prevalence and genetic mechanisms of antimicrobial resistance in Staphylococcus species: A multicentre report of the Indian council of medical research antimicrobial resistance surveillance network. Indian J Med Microbiol 2017;35:53-60.

12. Abimannan N, Sumathi G, Krishnarajasekhar OR, Sinha B, Krishnan P. Clonal clusters and virulence factors of methicillin-resistant Staphylococcus aureus: Evidence for community-acquired methicillinresistant Staphylococcus aureus infiltration into hospital settings in Chennai, South India. Indian J Med Microbiol 2019;37:326-36.

13. Singh L, Cariappa MP, Das NK. Drug sensitivity pattern of various Staphylococcus species isolated at a tertiary care hospital. Med J Armed Forces India 2016;72:S62-6.

14. Indian Network for Surveillance of Antimicrobial Resistance (INSAR) group, India. Methicillin resistant Staphylococcus aureus (MRSA) in India: Prevalence and susceptibility pattern. Indian J Med Res 2013;137:363-9.

15. Ghosh S, Banerjee M. Methicillin resistance and inducible clindamycin resistance in Staphylococcus aureus. Indian J Med Res 2016;143:362-4.

16. Mamtora D, Saseedharan S, Bhalekar P, Katakdhond S. Microbiological profile and antibiotic susceptibility pattern of gram-positive isolates at a tertiary care hospital. J Lab Physicians 2019;11:144-8.

17. Garoy EY, Gebreab YB, Achila OO, Tekeste DG, Kesete R, Ghirmay R, et al. Methicillin-resistant Staphylococcus aureus (MRSA): Prevalence and antimicrobial sensitivity pattern among patients a multicenter study in Asmara, Eritrea. Can J Infect Dis Med Microbiol 2019;2019:8321834.

18. Mama M, Aklilu A, Misgna K, Tadesse M, Alemayehu E. Methicillinand inducible clindamycin-resistant Staphylococcus aureus among patients with wound infection attending Arba Minch hospital, South Ethiopia. Int J Microbiol 2019:2965490.

19. Chinnambedu RS, Marimuthu RR, Sunil SS, Amrose P, Ramachandran V, Pachamuthu B. Changing antibiotic resistance profile of Staphylococcus aureus isolated from HIV patients (2012-2017) in Southern India. J Infect Public Health 2020;13:75-9.

20. Veeraraghavan B, Walia K. Antimicrobial susceptibility profile and resistance mechanisms of global antimicrobial resistance surveillance system (GLASS) priority pathogens from India. Indian J Med Res 2019;149:87-96. 